\title{
AN ANTICRACK IN A TRANSVERSELY ISOTROPIC SPACE
}

\author{
Andrzej KACZYŃSKI \\ Faculty of Mathematics and Information Science, Warsaw University of Technology, ul. Koszykowa 75, 00-662 Warszawa, Poland \\ akacz@mini.pw.edu.pl

\begin{abstract}
An absolutely rigid inclusion (anticrack) embedded in an unbound transversely isotropic elastic solid with the axis of elastic symmetry normal to the inclusion plane is considered. A general method of solving the anticrack problem is presented. Effective results have been achieved by constructing the appropriate harmonic potentials. With the use of the Fourier transform technique, the governing system of two-dimensional equations of Newtonian potential type for the stress jump functions on the opposite surfaces of the inclusion is obtained. For illustration, a complete solution to the problem of a penny-shaped anticrack under perpendicular tension at infinity is given and discussed from the point of view of material failure.
\end{abstract}

Key words: Three-Dimensional Elasticity, Transversely Isotropic Material, Plane Anticrack, Singular Integral Equations, Stress Singularity

\section{INTRODUCTION}

The study of the redistribution of stresses due to the presence of different kinds of defects in elastic bodies merit attention of specialists from many branches, such as geomechanics, metallurgy, material science. From the viewpoint of inhomogeneities in solids, cracks characterized by the displacement discontinuity and flat rigid inclusions (called anticracks) with the traction discontinuity are the two dangerous extremes. Although the crack problems have been studied extensively over the past 50 years, research on the corresponding anticrack problems have been rather limited and mainly concentrated on two-dimensional problems related to rigid line inclusions (see the monographs by Berezhnitskii et al. (1983) and Ting (1996)). The corresponding threedimensional analysis of a penny-shaped anticrack (absolutely rigid circular lamella) has been performed to a much lesser extent. Detailed account of elastostatic problems involving planar rigid inclusions embedded in homogeneous media is given in the studies by Kassir and Sih (1968), Selvadurai (1982), Silovanyuk (1984, 2000), Podil'chuk (1997), Rahman (1999, 2002), Chaudhuri (2003, 2012), Shodja and Ojaghnezhad (2007), and in the monographs by Mura (1981), Panasyuk et al. (1986), Khai (1993), Rogowski (2006), Kanaun and Levin (2008).

This paper is devoted to a three-dimensional static problem in linear elasticity theory of an infinite transversely isotropic body containing an arbitrarily shaped rigid inclusion lying in a plane of isotropy and subjected to some external loads. In Section 2 the basic equations with the potential representations of their general solution are reported. Besides, the anticrack problem is formulated. Section 3 presents a general method of solving the resulting boundary value problems. The governing 2-D singular integral equations are obtained in terms of the stress discontinuities across inclusion faces. As an illustration, a closed-form solution is given and discussed in Section 4 for a circular rigid inclusion subjected to tension at infinity. Finally, Section 5 concludes the paper.

\section{STATEMENT OF THE PROBLEM}

\subsection{Basic equations and general potential solutions}

The results of comprehensive research on the theory and applications of the mechanics of transversely isotropic elastic materials can be found in the book by Ding et al. (2006).

Referring to the rectangular Cartesian coordinate system $\left(x_{1}, x_{2}, x_{3}\right)$ denote at the point $\mathbf{x}=\left(x_{1}, x_{2}, x_{3}\right)$ the displacement vector by $\mathbf{u}=\left(u_{1}, u_{2}, u_{3}\right)$ and the stresses by $\sigma_{11}, \sigma_{12}$, $\sigma_{22}, \sigma_{13}, \sigma_{23}, \sigma_{33}$.

Throughout the considerations the following notations will be used: Latin subscripts always assume values 1, 2, 3 and the Greek ones 1, 2. The Einstein summation convention holds and subscripts preceded by a comma indicate partial differentiation with respect to the corresponding coordinates.

Consider a homogeneous transversely isotropic space assuming that the axis of elastic symmetry of the material coincides with the $x_{3}$-axis. Then the number of independent elastic stiffness constants (moduli) in the generalized Hooke's low reduces to five: $c_{11}, c_{12}, c_{13}, c_{33}, c_{44}$. The stress-displacement equations are the following:

$$
\begin{aligned}
& \sigma_{\alpha 3}=c_{44}\left(u_{\alpha, 3}+u_{3, \alpha}\right), \alpha=1,2 \\
& \sigma_{33}=c_{13} u_{\gamma, \gamma}+c_{33} u_{3,3} \\
& \sigma_{11}=c_{11} u_{1,1}+c_{12} u_{2,2}+c_{13} u_{3,3} \\
& \sigma_{22}=c_{12} u_{1,1}+c_{11} u_{2,2}+c_{13} u_{3,3} \\
& \sigma_{12}=0,5\left(c_{11}-c_{12}\right)\left(u_{1,2}+u_{2,1}\right)
\end{aligned}
$$

Inserting the above expressions into equilibrium equations in the absence of body forces

$$
\sigma_{j k, k}=0
$$


a governing system of three linear partial differential equations of second order with constant coefficients for displacements is obtained:

$$
\begin{aligned}
& 0,5\left(c_{11}+c_{12}\right) u_{\gamma, \gamma \alpha}+0,5\left(c_{11}-c_{12}\right) u_{\alpha, \gamma \gamma}+c_{44} u_{\alpha, 33}+ \\
& +\left(c_{13}+c_{44}\right) u_{3,3 \alpha}=0, \alpha=1,2 \\
& \left(c_{13}+c_{44}\right) u_{\gamma, \gamma 3}+c_{44} u_{3, \gamma \gamma}+c_{33} u_{3,33}=0
\end{aligned}
$$

According to the results obtained by Kaczyński (1993) with some modifications, the general solution of the above equations is found in terms of three potential functions $\varphi_{j} \equiv \varphi_{j}\left(x_{1}, x_{2}, z_{j}\right)$, $j=1,2,3$ with $z_{j}=t_{j} x_{3}$ which are harmonic in the appropriate coordinate systems $\left(x_{1}, x_{2}, z_{j}\right)$, i.e.

$\varphi_{j, \gamma \gamma}+\frac{\partial^{2} \varphi_{j}}{\partial z_{j}^{2}}=0, j=1,2,3 ;$ no sum on $j$

The form of displacement representation is dependent on the three material characterizing parameters $t_{j}$. The constant $t_{3}$ is given as:

$$
t_{3}=\sqrt{\left(c_{11}-c_{12}\right) / 2 c_{44}}>0
$$

while the constants $t_{\alpha}(\alpha=1,2)$ are the roots with positive real part of the following eigen-equation:

$$
c_{33} c_{44} t^{4}+\left[\left(c_{13}\right)^{2}+2 c_{13} c_{44}-c_{11} c_{33}\right] t^{2}+c_{11} c_{44}=0
$$

The analysis of modulus restrictions based on the positive definiteness of the stress energy leads to two cases (Ding et al.; 2006):

Case 1: $t_{1} \neq t_{2}$ when $A \neq 2 c_{44}$

$$
A_{-}>2 c_{44} \Rightarrow\left\{\begin{array}{l}
t_{1}=0,5\left(t_{+}-t_{-}\right)>0 \\
t_{2}=0,5\left(t_{+}+t_{-}\right)>0
\end{array}\right.
$$

or

$$
A_{-}<2 c_{44} \Rightarrow\left\{\begin{array}{l}
t_{1}=0,5\left(t_{+}-i t^{*}\right) \\
t_{2}=0,5\left(t_{+}+i t^{*}\right)
\end{array}, i^{2}=-1\right.
$$

where:

$$
\begin{aligned}
& A_{ \pm}=\sqrt{c_{11} c_{33}} \pm c_{13} \\
& t_{ \pm}=\sqrt{\left(A_{ \pm} \pm 2 c_{44}\right) A_{+} / c_{33} c_{44}} \\
& t^{*}=\sqrt{\left(2 c_{44}-A_{-}\right) A_{+} / c_{33} c_{44}}
\end{aligned}
$$

$$
\text { Case 2: } t_{1}=t_{2}
$$

$$
A=2 c_{44} \Rightarrow t_{1}=t_{2}=\sqrt[4]{c_{11} / c_{33}} \equiv t_{0}
$$

In the case when the material is isotropic it is found that:

$$
\begin{aligned}
& t_{0}=t_{3}=1 \\
& c_{11}=c_{33}=\lambda+2 \mu=\frac{2 \mu(1-v)}{1-2 v} \\
& c_{12}=c_{13}=\lambda=\frac{2 \mu \nu}{1-2 \nu}, \quad c_{44}=\mu
\end{aligned}
$$

Here, $\lambda, \mu$ are Lamé constants, and $v$ is Poisson's ratio.
For the sake of simplicity, hereinafter we proceed to consider the case with distinct eigenvalues (Case 1). Then the displacements and stresses can be represented in terms of potentials as follows:

$$
\begin{aligned}
& u_{\alpha}=\left(\varphi_{1}+\varphi_{2}\right)_{, \alpha}+\epsilon_{\alpha}^{\gamma} \varphi_{3, \gamma}, \alpha=1,2 \\
& u_{3}=m_{\gamma} t_{\gamma} \frac{\partial \varphi_{\gamma}}{\partial z_{\gamma}} \\
& \frac{\sigma_{3 \alpha}}{c_{44}}=\left(1+m_{\gamma}\right) t_{\gamma} \frac{\partial^{2} \varphi_{\gamma}}{\partial z_{\gamma} \partial x_{\alpha}}+\epsilon_{\alpha}^{\gamma} t_{3} \frac{\partial^{2} \varphi_{3}}{\partial z_{3} \partial x_{\gamma}} \\
& \frac{\sigma_{33}}{c_{44}}=\left(1+m_{\gamma}\right) \frac{\partial^{2} \varphi_{\gamma}}{\partial z_{\gamma}^{2}} \\
& \sigma_{11}=-c_{44}\left(1+m_{\gamma}\right) t_{\gamma}^{2} \frac{\partial^{2} \varphi_{\gamma}}{\partial z_{\gamma}^{2}}+ \\
&-\left(c_{11}-c_{12}\right)\left[\left(\varphi_{1}+\varphi_{2}\right)_{, 22}+\varphi_{3,12}\right] \\
& \sigma_{22}=-c_{44}\left(1+m_{\gamma}\right) t_{\gamma}^{2} \frac{\partial^{2} \varphi_{\gamma}}{\partial z_{\gamma}^{2}}+ \\
&-\left(c_{11}-c_{12}\right)\left[\left(\varphi_{1}+\varphi_{2}\right)_{11}-\varphi_{3,12}\right]
\end{aligned}
$$

Here $\epsilon_{2}^{1}=1, \epsilon_{1}^{2}=-1, \epsilon_{1}^{1}=\epsilon_{2}^{2}=0$ and the constants $m_{1}, m_{2}$ related to $t_{1}, t_{2}$ are defined as:

$$
m_{\alpha}=\frac{c_{11}-t_{\alpha}^{2} c_{44}}{\left(c_{13}+c_{44}\right) t_{\alpha}^{2}}=\frac{c_{13}+c_{44}}{t_{\alpha}^{2} c_{33}-c_{44}}, \alpha=1,2
$$

It is worth noting that:

$$
\begin{aligned}
& \left\{\begin{array}{l}
c_{33} m_{\alpha} t_{\alpha}^{2}-c_{13}=c_{44}\left(1+m_{\alpha}\right) \\
c_{11}-c_{13} m_{\alpha} t_{\alpha}^{2}=c_{44} t_{\alpha}^{2}\left(1+m_{\alpha}\right)
\end{array}, \alpha=1 \text { or } 2, \text { no sum on } \alpha\right. \\
& m_{1} m_{2}=1 \\
& t_{1} t_{2}=\sqrt{c_{11} / c_{33}} \\
& \left(t_{1}\right)^{2}+\left(t_{2}\right)^{2}=\left(c_{11} c_{33}-c_{13}^{2}-2 c_{13} c_{44}\right) / c_{33} c_{44}
\end{aligned}
$$

\subsection{Formulation}

Consider a transversely isotropic space with the axis of symmetry as the $x_{3}$-axis and the isotropic plane as the $x_{1}, x_{2}$ - plane. Suppose that this body is weakened by a rigid-sheet like inclusion (anticrack) occupying a certain domain $S$ with a smooth boundary at the plane $x_{3}=0$ and subjected to some external loads.

It is known that an anticrack problem can be regarded as the superposition of two problems. One (labelled by the subscript 0) is no inclusion problem with the given applied loadings and the other is the perturbed problem in which the displacements along the anticrack $S$ are prescribed as the negative of those generated in the first problem. Thus, the total displacement-stress field (denoted by $u_{j}^{(t)}$ and $\sigma_{j k}^{(t)}$ ) can be expressed as: 
$u_{j}^{(t)}=u_{j}^{(0)}+u_{j}, \quad \sigma_{j k}^{(t)}=\sigma_{j k}^{(0)}+\sigma_{j k}$

Moreover, we assume that $u_{j}^{(0)}$ and $\left.\sigma_{j k}^{(0)}\right)$ are known from the solution to the 0-problem. As a matter of fact, only the values of displacements $\quad \bar{u}_{j}^{(0)} \equiv u_{j}^{(0)}\left(x_{1}, x_{2}, 0\right),\left(x_{1}, x_{2}\right) \in S \quad$ are needed in the subsequent analysis.

Next, we concentrate attention on the non-trivial perturbed problem, solution to which tends to zero at infinity and satisfies the necessary boundary conditions on $S$ :

$u_{1}=-\bar{u}_{1}^{(0)}+\varepsilon_{1}-\omega_{3} x_{2}$

$u_{2}=-\bar{u}_{2}^{(0)}+\varepsilon_{2}+\omega_{3} x_{1}$

$u_{3}=-\bar{u}_{3}^{(0)}+\varepsilon_{3}-\omega_{2} x_{1}+\omega_{1} x_{2}$

in which also the unknown small displacements $\varepsilon_{j}$ and rotations $\omega_{j}$ are included to describe a motion of the inclusion as a rigid unit. These parameters will be determined later in solving the problem in hand from the equilibrium conditions of the anticrack (no resultant forces and zero-moments).

Now we proceed to the reduction of the above anticrack problem to some mixed boundary-value problems of potential theory related to the one of a half-space (say, $x_{3} \geq 0$ ). From the relevant symmetry properties about the plane $x_{3}=0$ follows the division of the problem into antisymmetric $(A)$ and symmetric ones (B).

In the antisymmetric part $(A)$ of the problem, the mixed conditions on the plane of antisymmetry may be written as

$u_{3}=-\bar{u}_{3}^{(0)}+\varepsilon_{3}-\omega_{2} x_{1}+\omega_{1} x_{2},\left(x_{1}, x_{2}\right) \in S$

$u_{1}=u_{2}=0,\left(x_{1}, x_{2}\right) \in R^{2}$

$\sigma_{33}=0,\left(x_{1}, x_{2}\right) \in R^{2}-S$

and are supplemented by the corresponding equilibrium conditions to determine the unknown parameters $\varepsilon_{3}, \omega_{\alpha}(\alpha=$ $1,2)$ :

$\iint_{S}\left[\sigma_{33}\left(x_{1}, x_{2}, 0^{+}\right)-\sigma_{33}\left(x_{1}, x_{2}, 0^{-}\right)\right] d x_{1} d x_{2}=0$
$\iint_{S} x_{3-\alpha}\left[\sigma_{33}\left(x_{1}, x_{2}, 0^{+}\right)-\sigma_{33}\left(x_{1}, x_{2}, 0^{-}\right)\right] d x_{1} d x_{2}=0$

The symmetric part $(B)$ of the problem leads to the following mixed conditions

$u_{1}=-\bar{u}_{1}^{(0)}+\varepsilon_{1}-\omega_{3} x_{2},\left(x_{1}, x_{2}\right) \in S$

$u_{2}=-\bar{u}_{2}^{(0)}+\varepsilon_{2}+\omega_{3} x_{1},\left(x_{1}, x_{2}\right) \in S$

$u_{3}=0,\left(x_{1}, x_{2}\right) \in R^{2}$

$\sigma_{31}=\sigma_{32}=0,\left(x_{1}, x_{2}\right) \in R^{2}-S$

and additional conditions to determine the unknown parameters $\varepsilon_{\alpha}(\alpha=1,2), \omega_{3}$ :

$$
\begin{aligned}
& \iint_{S}\left[\sigma_{3 \alpha}\left(x_{1}, x_{2}, 0^{+}\right)-\sigma_{3 \alpha}\left(x_{1}, x_{2}, 0^{-}\right)\right] d x_{1} d x_{2}=0 \\
& \iint_{S} x_{2}\left[\sigma_{31}\left(x_{1}, x_{2}, 0^{+}\right)-\sigma_{31}\left(x_{1}, x_{2}, 0^{-}\right)\right] d x_{1} d x_{2}+ \\
& -\iint_{S} x_{1}\left[\sigma_{32}\left(x_{1}, x_{2}, 0^{+}\right)-\sigma_{32}\left(x_{1}, x_{2}, 0^{-}\right)\right] d x_{1} d x_{2}=0
\end{aligned}
$$

In addition to these boundary conditions, the displacement and stress fields must vanish at infinity.

\section{SOLUTION METHOD}

The above-mentioned problems $(\mathrm{A})$ and $(\mathrm{B})$ are now reducing to some mixed boundary-value problems of potential theory by constructing the potentials functions well suited to the boundary conditions (30)-(32) and (35)-(38), respectively. Further, an integral equation formulation is given for these problems.

\subsection{Antisymmetric problem}

It is expedient to choose in the displacement-stress representations (17)-(23) only one harmonic function $f\left(x_{1}, x_{2}, x_{3}\right)$ such that:

$$
\begin{aligned}
& \varphi_{1}\left(x_{1}, x_{2}, z_{1}\right) \equiv-f\left(x_{1}, x_{2}, z_{1}\right) \\
& \varphi_{2}\left(x_{1}, x_{2}, z_{2}\right) \equiv f\left(x_{1}, x_{2}, z_{2}\right) \\
& \varphi_{3}\left(x_{1}, x_{2}, z_{3}\right) \equiv 0
\end{aligned}
$$

Then the corresponding displacement and stress components become:

$$
\begin{aligned}
& u_{\alpha}=(-1)^{\beta}\left[f\left(x_{1}, x_{2}, z_{\alpha}\right)\right]_{, \beta}, \alpha=1,2 \\
& u_{3}=(-1)^{\beta} m_{\beta} t_{\beta} \frac{\partial f\left(x_{1}, x_{2}, z_{\beta}\right)}{\partial z_{\beta}}
\end{aligned}
$$$$
\frac{\sigma_{3 \alpha}}{c_{44}}=(-1)^{\beta}\left(1+m_{\beta}\right) t_{\beta} \frac{\partial^{2} f\left(x_{1}, x_{2}, z_{\beta}\right)}{\partial z_{\beta} \partial x_{\alpha}}, \alpha=1,2
$$

$\frac{\sigma_{33}}{c_{44}}=(-1)^{\beta}\left(1+m_{\beta}\right) \frac{\partial^{2} f\left(x_{1}, x_{2}, z_{\beta}\right)}{\partial z_{\beta}^{2}}$

$\sigma_{11}=-c_{44}(-1)^{\beta}\left(1+m_{\beta}\right) t_{\beta}^{2} \frac{\partial^{2} f}{\partial z_{\beta}^{2}}+$

$$
-\left(c_{11}-c_{12}\right)(-1)^{\beta}\left[f\left(x_{1}, x_{2}, z_{\beta}\right)\right], 22
$$

$$
\begin{aligned}
\sigma_{22}= & -c_{44}(-1)^{\beta}\left(1+m_{\beta}\right) t_{\beta}^{2} \frac{\partial^{2} f}{\partial z_{\beta}^{2}}+ \\
& -\left(c_{11}-c_{12}\right)(-1)^{\beta}\left[f\left(x_{1}, x_{2}, z_{\beta}\right)\right]_{, 11}
\end{aligned}
$$

$\sigma_{12}=\left(c_{11}-c_{12}\right)(-1)^{\beta}\left[f\left(x_{1}, x_{2}, z \beta\right)\right]_{12}$

In the limit as $x_{3} \rightarrow 0^{\mp}$, the above equations reduce to: 


$$
\begin{aligned}
& u_{1}=u_{2}=0 \\
& u_{3}=\left(m_{2} t_{2}-m_{1} t_{1}\right)\left[f_{, 3}\left(x_{1}, x_{2}, x_{3}\right)\right]_{X_{3}}=0 \\
& \frac{\sigma_{3 \alpha}}{c_{44}}=\left(m_{2} t_{2}-m_{1} t_{1}+t_{2}-t_{1}\right)\left[f_{, 3 \alpha}\left(x_{1}, x_{2}, x_{3}\right)\right]_{X_{3}=0} \\
& \frac{\sigma_{33}^{ \pm}}{c_{44}}=\left(m_{2}-m_{1}\right)\left[f_{, 33}\left(x_{1}, x_{2}, x_{3}\right)\right]_{x_{3}=0^{ \pm}} \\
& \sigma_{11}^{ \pm}=\sigma_{22}^{ \pm}=-c_{44}\left[m_{2}\left(t_{2}\right)^{2}-m_{1}\left(t_{1}\right)^{2}+\left(t_{2}\right)^{2}-\left(t_{1}\right)^{2}\right] \\
& \cdot\left[f_{, 33}\left(x_{1}, x_{2}, x_{3}\right)\right]_{x_{3}=0^{ \pm}}
\end{aligned}
$$

$\sigma_{12}=0$

A glance at equations (37)-(39) reveals that the potential $f\left(x_{1}, x_{2}, x_{3}\right)$ is governed by:

- for $\left(x_{1}, x_{2}\right) \in S$

$$
\begin{aligned}
& {\left[f_{, 3}\left(x_{1}, x_{2}, x_{3}\right)\right]_{X_{3}=0^{+}}=\frac{-\bar{u}_{3}^{(0)}+\varepsilon_{3}-\omega_{2} x_{1}+\omega_{1} x_{2}}{m_{2} t_{2}-m_{1} t_{1}}} \\
& - \text { for }\left(x_{1}, x_{2}\right) \in R^{2}-S \\
& {\left[f_{, 33}\left(x_{1}, x_{2}, x_{3}\right)\right]_{X_{3}=0^{+}}=0}
\end{aligned}
$$

\subsection{Symmetric problem}

This problem is substantially more complex (see the corresponding skew-symmetrical crack problem considered in Kassir and $\operatorname{Sih}(1975))$. We choose the potential functions in the general representation (16)-(22) such that:

$$
\begin{aligned}
\varphi_{1} & =-\frac{m_{2} t_{2}}{m_{2} t_{2}-m_{1} t_{1}}\left(G_{1,1}+H_{1,2}\right) \equiv-\frac{m_{2} t_{2}}{m_{2} t_{2}-m_{1} t_{1}} F_{1} \\
\varphi_{2} & =\frac{m_{1} t_{1}}{m_{2} t_{2}-m_{1} t_{1}}\left(G_{2,1}+H_{2,2}\right) \equiv \frac{m_{1} t_{1}}{m_{2} t_{2}-m_{1} t_{1}} F_{2} \\
\varphi_{3} & =G_{3,2}-H_{3,1} \equiv F_{3}
\end{aligned}
$$

where $G_{j} \equiv G\left(x_{1}, x_{2}, z_{j}\right)$ and $H_{j} \equiv H\left(x_{1}, x_{2}, z_{j}\right), j=1,2,3$ are harmonic functions in the corresponding systems of coordinates. Then, the corresponding displacement-stress field becomes:

$$
\begin{aligned}
& u_{\alpha}=\frac{\left[m_{1} t_{1} F_{2}-m_{2} t_{2} F_{1}\right], \alpha}{m_{2} t_{2}-m_{1} t_{1}}+\epsilon_{\alpha}^{\gamma} F_{3, \gamma}, \alpha=1,2 \\
& u_{3}=\frac{t_{1} t_{2}}{m_{2} t_{2}-m_{1} t_{1}}\left(\frac{\partial F_{2}}{\partial z_{2}}-\frac{\partial F_{1}}{\partial z_{1}}\right) \\
& \frac{\sigma_{3 \alpha}}{c_{44}}=\frac{t_{1} t_{2}}{m_{2} t_{2}-m_{1} t_{1}}\left[\left(1+m_{1}\right) \frac{\partial F_{2}}{\partial z_{2}}-\left(1+m_{2}\right) \frac{\partial F_{1}}{\partial z_{1}}\right], \alpha \\
& +\epsilon_{\alpha}^{\gamma} t_{3} \frac{\partial^{2} F_{3}}{\partial z_{3} \partial x_{\gamma}}, \alpha=1,2 \\
& \frac{\sigma_{33}}{c_{44}}=\frac{1}{m_{2} t_{2}-m_{1} t_{1}}\left[t_{1}\left(1+m_{1}\right) \frac{\partial^{2} F_{2}}{\partial z_{2}^{2}}-t_{2}\left(1+m_{2}\right) \frac{\partial^{2} F_{1}}{\partial z_{1}^{2}}\right]
\end{aligned}
$$

$$
\begin{array}{r}
\sigma_{11}=\frac{-c_{44} t_{1} t_{2}}{m_{2} t_{2}-m_{1} t_{1}}\left[t_{2}\left(1+m_{1}\right) \frac{\partial^{2} F_{2}}{\partial z_{2}^{2}}-t_{1}\left(1+m_{2}\right) \frac{\partial^{2} F_{1}}{\partial z_{1}^{2}}\right] \\
-\left(c_{11}-c_{12}\right)\left[\frac{\left(m_{1} t_{1} F_{2}-m_{2} t_{2} F_{1}\right), 22}{m_{2} t_{2}-m_{1} t_{1}}+F_{3}, 12\right] \\
\sigma_{22}=\frac{-c_{44} t_{1} t_{2}}{m_{2} t_{2}-m_{1} t_{1}}\left[t_{2}\left(1+m_{1}\right) \frac{\partial^{2} F_{2}}{\partial z_{2}^{2}}-t_{1}\left(1+m_{2}\right) \frac{\partial^{2} F_{1}}{\partial z_{1}^{2}}\right] \\
-\left(c_{11}-c_{12}\right)\left[\frac{\left(m_{1} t_{1} F_{2}-m_{2} t_{2} F_{1}\right), 11}{m_{2} t_{2}-m_{1} t_{1}}-F_{3}, 12\right] \\
\frac{\sigma_{12}}{c_{11}-c_{12}}=\frac{\left(m_{1} t_{1} F_{2}-m_{2} t_{2} F_{1}\right), 12}{m_{2} t_{2}-m_{1} t_{1}}+0,5\left(F_{3}, 11-F_{3}, 22\right)
\end{array}
$$

It is seen that the above expressions simplify across the plane $x_{3}=0$ since the subscripts $j$ can be dropped for $G_{j}$ and $H_{j}$, so we get $F_{1}=F_{2}=G_{, 1}+H_{, 2}, F_{3}=G_{, 2}-H_{, 1}$. Introducing the notation:

$$
\begin{aligned}
& g\left(x_{1}, x_{2}, x_{3}\right)=\frac{\partial G\left(x_{1}, x_{2}, x_{3}\right)}{\partial x_{3}} \\
& h\left(x_{1}, x_{2}, x_{3}\right)=\frac{\partial H\left(x_{1}, x_{2}, x_{3}\right)}{\partial x_{3}}
\end{aligned}
$$

the displacements and stresses in the plane of symmetry are found as:

$$
\begin{aligned}
& u_{1}=\left[g, 3\left(x_{1}, x_{2}, x_{3}\right)\right]_{X_{3}=0} \\
& u_{2}=\left[h, 3\left(x_{1}, x_{2}, x_{3}\right)\right]_{X_{3}=0} \\
& u_{3}=0 \\
& \frac{\sigma_{31}^{ \pm}}{c_{44}}=C^{*}[g, 33+\kappa(g, 22-h, 12)]_{X_{3}=0^{ \pm}} \\
& \frac{\sigma_{32}^{ \pm}}{c_{44}}=C^{*}[h, 33+\kappa(h, 11-g, 12)]_{X_{3}=0^{ \pm}} \\
& \frac{\sigma_{33}}{c_{44}}=-D^{\star}[g, 31+h, 32]_{X_{3}=0} \\
& \sigma_{11}=E^{*}[g, 31+h, 32]_{X_{3}=0}-\left(c_{11}-c_{12}\right)[h, 32]_{X_{3}=0} \\
& \sigma_{22}=E^{*}[g, 31+h, 32]_{X_{3}=0}-\left(c_{11}-c_{12}\right)[g, 31]_{X_{3}=0} \\
& \sigma_{12}=0,5\left(c_{11}-c_{12}\right)[g, 32+h, 31]_{X_{3}=0}
\end{aligned}
$$

where:

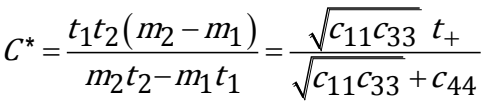

$$
\begin{aligned}
& \kappa=1-\frac{t_{3}}{C^{*}} \\
& D^{*}=\frac{A}{\sqrt{c_{11} c_{33}}+c_{44}}, \quad E^{*}=\sqrt{\frac{c_{11}}{c_{33}}} \frac{A_{-}\left(A_{+}+c_{44}\right)}{\sqrt{c_{11} c_{33}}+c_{44}}
\end{aligned}
$$

It follows immediately from the boundary conditions (35)-(38) that the unknown potentials $g$ and $h$ can be determined from: 


$$
\begin{aligned}
& \left.\begin{array}{l}
{\left[g, 3\left(x_{1}, x_{2}, x_{3}\right)\right]_{X_{3}=0^{+}}=-\bar{u}_{1}^{(0)}+\varepsilon_{1}-\omega_{3} x_{2}} \\
{\left[h_{, 3}\left(x_{1}, x_{2}, x_{3}\right)\right]_{X_{3}=0^{+}}=-\bar{u}_{2}^{(0)}+\varepsilon_{2}+\omega_{3} x_{1}}
\end{array}\right\}\left(x_{1}, x_{2}\right) \in S \\
& \left.\begin{array}{l}
{[g, 33+\kappa(g, 22-h, 12)]_{X_{3}=0^{+}}=0} \\
{\left[h, 33+\kappa\left(h, 11^{-} g, 12\right)\right]_{X_{3}=0^{+}}=0}
\end{array}\right\}\left(x_{1}, x_{2}\right) \in R^{2}-S
\end{aligned}
$$

It is easy to seen from a comparison of the above conditions that the formulation given by Eqs. (74) and (75) is inverse to that for the crack problem involving shear tractions (Kassir and Sih, 1975).

To obtain integral equations, the two-dimensional Fourier technique will be used (see the method developed by Kaczyński (1999)). The space harmonic functions $f, g$ and $h$ are represented by Fourier's integrals (Sneddon, 1972):

$$
\left[\begin{array}{c}
f(\mathbf{x}) \\
g(\mathbf{x}) \\
h(\mathbf{x})
\end{array}\right]=\iint_{S} \frac{\exp \left[-x_{3}|\xi|+i\left(x_{\alpha} \xi_{\alpha}\right)\right]}{|\xi|^{2}}\left[\begin{array}{l}
A_{f}(\xi) \\
A_{g}(\xi) \\
A_{h}(\xi)
\end{array}\right] d S_{\xi}
$$

where $\xi=\left(\xi_{1}, \xi_{2}\right) \in S,|\xi|=\sqrt{\left(\xi_{1}\right)^{2}+\left(\xi_{2}\right)^{2}}$.

From the boundary condition (56), in view of (52), one finds immediately that:

$A_{f}\left(\xi_{1}, \xi_{2}\right)=\frac{1}{4 \pi^{2} c_{44}\left(m_{2}-m_{1}\right)} \iint_{S} \sigma_{33}^{+}\left(\eta_{1}, \eta_{2}\right) \exp \left[-i\left(\eta_{\alpha} \xi_{\alpha}\right)\right] d \eta_{1} d \eta_{2}(77)$

Accordingly, the conditions in equation (75) yield a system for the remaining unknown functions $A_{g}(\xi)$ and $A_{h}(\xi)$ :

$$
\begin{aligned}
& {\left[\begin{array}{cc}
1-\frac{\kappa\left(\xi_{2}\right)^{2}}{|\xi|^{2}} & \frac{\kappa \xi_{1} \xi_{2}}{|\xi|^{2}} \\
\frac{\kappa \xi_{1} \xi_{2}}{|\xi|^{2}} & 1-\frac{\kappa\left(\xi_{1}\right)^{2}}{|\xi|^{2}}
\end{array}\right]\left[\begin{array}{l}
A_{g}\left(\xi_{1}, \xi_{2}\right) \\
A_{h}\left(\xi_{1}, \xi_{2}\right)
\end{array}\right]=} \\
& =\frac{1}{4 \pi^{2} c_{44} C^{*}} \iint_{S}\left[\begin{array}{c}
\sigma_{31}^{+}\left(\eta_{1}, \eta_{2}\right) \\
\sigma_{32}^{+}\left(\eta_{1}, \eta_{2}\right)
\end{array}\right] \exp \left[-i\left(\eta_{\alpha} \xi_{\alpha}\right)\right] d \eta_{1} d \eta_{2}
\end{aligned}
$$

which has the solution:

$$
\begin{array}{r}
4 \pi^{2} c_{44} C^{*}(1-\kappa)\left[\begin{array}{l}
A_{g}(\xi) \\
A_{h}(\xi)
\end{array}\right]=\left[\begin{array}{cc}
1-\frac{\kappa\left(\xi_{1}\right)^{2}}{|\xi|^{2}} & -\frac{\kappa \xi_{1} \xi_{2}}{|\xi|^{2}} \\
-\frac{\kappa \xi_{1} \xi_{2}}{|\xi|^{2}} & 1-\frac{\kappa\left(\xi_{2}\right)^{2}}{|\xi|^{2}}
\end{array}\right] . \\
\cdot \iint_{S}\left[\begin{array}{l}
\sigma_{31}^{+}\left(\eta_{1}, \eta_{2}\right) \\
\sigma_{32}^{+}\left(\eta_{1}, \eta_{2}\right)
\end{array}\right] \exp \left[-i\left(\eta_{\alpha} \xi_{\alpha}\right)\right] d \eta_{1} d \eta_{2}
\end{array}
$$

Equations (77) and (79) may be substituted into (76) to give:

$$
2 \pi c_{44}\left(m_{2}-m_{1}\right) f_{, 3}(\mathbf{x})=-\iint_{S} \frac{\sigma_{33}^{+}(\xi)}{|\mathbf{x}-\xi|} d S_{\xi}
$$

$$
\begin{aligned}
-2 \pi t_{3} c_{44} g, 3(\mathbf{x}) & =\iint_{S} \frac{\sigma_{31}^{+}(\xi)}{|\mathbf{x}-\xi|} d S_{\xi}-\kappa \iint_{S} \frac{\sigma_{31}^{+}(\xi)\left(x_{2}-\xi_{2}\right)^{2}}{|\mathbf{x}-\xi|^{3}} d S_{\xi} \\
& +\kappa \iint_{S} \frac{\sigma_{32}^{+}(\xi)\left(x_{1}-\xi_{1}\right)\left(x_{2}-\xi_{2}\right)}{|\mathbf{x}-\xi|^{3}} d S_{\xi} \\
-2 \pi t_{3} c_{44} h_{, 3}(\mathbf{x}) & =\iint_{S} \frac{\sigma_{32}^{+}(\xi)}{|\mathbf{x}-\xi|} d S_{\xi}-\kappa \iint_{S} \frac{\sigma_{32}^{+}(\xi)\left(x_{1}-\xi_{1}\right)^{2}}{|\mathbf{x}-\xi|^{3}} d S_{\xi} \\
+\kappa & \iint_{S} \frac{\sigma_{31}^{+}(\xi)\left(x_{1}-\xi_{1}\right)\left(x_{2}-\xi_{2}\right)}{|\mathbf{x}-\xi|^{3}} d S_{\xi}
\end{aligned}
$$

where $\sigma_{3 j}^{+}(\xi)=\sigma_{3 j}\left(\xi_{1}, \xi_{2}, 0^{+}\right), \xi=\left(\xi_{1}, \xi_{2}, 0\right) \in S$ and $\mid \mathbf{x}-$ $\xi \mid$ is a distance between the field point $\mathbf{x}=\left(x_{1}, x_{2}, x_{3}\right)$ and the integration point $\xi=\left(\xi_{1}, \xi_{2}, 0\right)$. Moreover, in deriving the above expressions, use has been made of the following integrals (Erdelyi, 1954):

$$
\begin{aligned}
& \iint_{R^{2}} \frac{\exp i\left[\left(x_{\alpha}-\eta_{\alpha}\right) \xi_{\alpha}\right]}{|\xi|} d \xi_{1} d \xi_{2}=\frac{2 \pi}{\sqrt{\left(x_{1}-\eta_{1}\right)^{2}+\left(x_{2}-\eta_{2}\right)^{2}}} \\
& \iint_{R^{2}} \frac{\exp i\left[\left(x_{\alpha}-\eta_{\alpha}\right) \xi_{\alpha}\right]\left(\xi_{1}\right)^{2}}{|\xi|^{3}} d \xi_{1} d \xi_{2}=\frac{2 \pi\left(x_{2}-\eta_{2}\right)}{\left(\sqrt{\left(x_{1}-\eta_{1}\right)^{2}+\left(x_{2}-\eta_{2}\right)^{2}}\right)^{3}} \\
& \iint_{R^{2}} \frac{\exp i\left[\left(x_{\alpha}-\eta_{\alpha}\right) \xi_{\alpha}\right]\left(\xi_{2}\right)^{2}}{|\xi|^{3}} d \xi_{1} d \xi_{2}=\frac{2 \pi\left(x_{1}-\eta_{1}\right)}{\left(\sqrt{\left(x_{1}-\eta_{1}\right)^{2}+\left(x_{2}-\eta_{2}\right)^{2}}\right)^{3}} \\
& \iint_{R^{2}} \frac{\exp i\left[\left(x_{\alpha}-\eta_{\alpha}\right) \xi_{\alpha}\right] \xi_{1} \xi_{2}}{|\xi|^{3}} d \xi_{1} d \xi_{2}=\frac{-2 \pi\left(x_{1}-\eta_{1}\right)\left(x_{2}-\eta_{2}\right)}{\left(\sqrt{\left(x_{1}-\eta_{1}\right)^{2}+\left(x_{2}-\eta_{2}\right)^{2}}\right)^{3}}
\end{aligned}
$$

Now enforcing the displacement boundary conditions (55) and (74), we arrive at the governing singular integral equations of Newtonian potential type to determine the interface stresses $\sigma_{3 j}^{+} \mid s, j=1,2,3$ :

$A \iint_{S} \frac{\sigma_{33}^{+}\left(\xi_{1}, \xi_{2}\right) d \xi_{1} d \xi_{2}}{\sqrt{\left(x_{1}-\xi_{1}\right)^{2}+\left(x_{2}-\xi_{2}\right)^{2}}}=\bar{u}_{3}^{(0)}-\varepsilon_{3}+\omega_{2} x_{1}-\omega_{1} x_{2}$

$$
B \iint_{S}\left\{\frac{\sigma_{31}^{+}\left(\xi_{1}, \xi_{2}\right)}{\sqrt{\left(x_{1}-\xi_{1}\right)^{2}+\left(x_{2}-\xi_{2}\right)^{2}}}\left[1-\kappa \frac{\left(x_{2}-\xi_{2}\right)^{2}}{\left(x_{1}-\xi_{1}\right)^{2}+\left(x_{2}-\xi_{2}\right)^{2}}\right]+\right.
$$$$
\left.+\kappa \frac{\sigma_{32}^{+}\left(\xi_{1}, \xi_{2}\right)\left(x_{1}-\xi_{1}\right)\left(x_{2}-\xi_{2}\right)}{\left(\sqrt{\left(x_{1}-\xi_{1}\right)^{2}+\left(x_{2}-\xi_{2}\right)^{2}}\right)^{3}}\right\} d \xi_{1} d \xi_{2}=\bar{u}_{1}^{(0)}-\varepsilon_{1}+\omega_{3} x_{2}
$$ 


$$
\begin{aligned}
& B \int\left\{\int _ { S } \left\{\frac{\sigma_{32}^{+}\left(\xi_{1}, \xi_{2}\right)}{\sqrt{\left(x_{1}-\xi_{1}\right)^{2}+\left(x_{2}-\xi_{2}\right)^{2}}}\left[1-\kappa \frac{\left(x_{1}-\xi_{1}\right)^{2}}{\left(x_{1}-\xi_{1}\right)^{2}+\left(x_{2}-\xi_{2}\right)^{2}}\right]+\right.\right. \\
& \left.+\kappa \frac{\sigma_{31}^{+}\left(\xi_{1}, \xi_{2}\right)\left(x_{1}-\xi_{1}\right)\left(x_{2}-\xi_{2}\right)}{\left(\sqrt{\left(x_{1}-\xi_{1}\right)^{2}+\left(x_{2}-\xi_{2}\right)^{2}}\right)^{3}}\right\} d \xi_{1} d \xi_{2}=\bar{u}_{2}^{(0)}-\varepsilon_{2}-\omega_{3} x_{1}
\end{aligned}
$$

in which $\kappa$ is given by Eqs. (73), and the material constants $A, B$ are:

$$
\begin{aligned}
& A=\frac{m_{2} t_{2}-m_{1} t_{1}}{2 \pi c_{44}\left(m_{2}-m_{1}\right)}=\frac{\sqrt{c_{11} c_{33}}+c_{44}}{2 \pi c_{44} c_{33} t_{+}} \\
& B=\frac{1}{2 \pi c_{44} t_{3}}=\frac{1}{\pi \sqrt{2 c_{44}\left(c_{11}-c_{12}\right)}}
\end{aligned}
$$

It is worth mentioning that the results obtained can be used in the case of isotropic material (see the relations (16)). Then the constants in the governing equations become:

$$
\begin{aligned}
& \kappa=\frac{\lambda+\mu}{2(\lambda+2 \mu)}=\frac{1}{4(1-v)} \\
& A=\frac{\lambda+3 \mu}{4 \pi \mu(\lambda+2 \mu)}=\frac{3-4 v}{8 \pi \mu(1-v)} \\
& B=\frac{1}{2 \pi \mu}
\end{aligned}
$$

which are in agreement with those obtained by Silovanyuk (1984).

After the stresses $\sigma_{3 j}$ acting on the inclusion side $S^{+}$have been determined from the solution of the above integral equations, the full-space elastic field can be calculated from the harmonic potentials $f, g, h$ via relations (80)-(83) with the use of Eqs. (42)-(48) and (57)-(64). For completeness, the kinematical parameters of the inclusion $\varepsilon_{j}$ and $\omega_{j}$ can be found from the conditions (33), (39) and (34), (40), respectively.

For an arbitrary simply connected domain $S$ bounded by a smooth contour the governing equations can be solved by an analytic-numerical method developed in Kit et al. (1989). Explicit solutions are possible for elliptical shapes of $S$ by assuming polynomial right-hand sides. The typical case of a circular anticrack in a uniform normal tension at infinity will be considered in the next section.

\section{EXAMPLE}

The stress state of a transversely isotropic space containing in the plane of isotropy a sealed circular rigid inclusion (anticrack) $s=\left\{\left(x_{1}, x_{2}, 0\right): r \equiv \sqrt{\left(x_{1}\right)^{2}+\left(x_{2}\right)^{2}} \leq a\right\}$ is investigated by assuming that:

$$
\sigma_{33}^{(t)}(\infty)=\sigma_{3}=\text { const. }>0, \quad \sigma_{31}^{(t)}(\infty)=\sigma_{32}^{(t)}(\infty)=0
$$

The displacement solution to the basic equations (7) and (8) of 0 -problem with conditions (89) is readily obtained as:

$$
\begin{aligned}
& u_{\alpha}^{(0)}\left(x_{1}, x_{2}, x_{3}\right)=-D \sigma_{3} x_{\alpha}, \alpha=1,2 \\
& u_{3}^{(0)}\left(x_{1}, x_{2}, x_{3}\right)=D_{3} \sigma_{3} x_{3}
\end{aligned}
$$

Where:

$$
\begin{aligned}
& D=\frac{c_{13}}{c_{33}\left(c_{11}+c_{12}\right)-2\left(c_{13}\right)^{2}} \\
& D_{3}=\frac{c_{11}+c_{12}}{c_{33}\left(c_{11}+c_{12}\right)-2\left(c_{13}\right)^{2}}
\end{aligned}
$$

Now invoking the displacement boundary conditions (see Eqs. (27)-(29)), we are dealing with the symmetric part of the perturbed problem described by Eqs. (35)-(40) in which

$$
\bar{u}_{\alpha}\left(x_{1}, x_{2}\right)=-D \sigma_{3} x_{\alpha}, \alpha=1,2
$$

The exact analytical solution of the governing system (85) and (86) with the RHS given by (92) is achieved if we take the unknown stresses in the form

$$
\sigma_{3 \alpha}^{+}\left(x_{1}, x_{2}\right)=\frac{b_{0 \alpha}+b_{1 \alpha} x_{1}+b_{2 \alpha} x_{2}}{\sqrt{a^{2}-r^{2}}}, \alpha=1,2
$$

where $b_{j \alpha}, j=0,1,2, \alpha=1,2$ are unknown constants.

Substituting these expressions into Eqs. (85) and (86), and next using the formulas for resulting integrals given by Vorovich et al. (1974), the equality of two polynomials of the first order is obtained. Equating the terms of the left and right hands with the same powers of $x_{1}$ and $x_{2}$ yields a system of linear algebraic equations, solving which we get the following formulas for the unknown coefficients $b_{j \alpha}$ :

$$
\begin{aligned}
& b_{0 \alpha}=-\frac{4 c_{44} t_{3}}{\pi(2-\kappa)} \varepsilon_{\alpha}, \alpha=1,2 \\
& b_{12}=-b_{21}=\frac{4}{\pi} c_{44} t_{3} \omega_{3} \\
& b_{11}=b_{22}=-\frac{4}{\pi} c_{44} D C^{*} \sigma_{3}
\end{aligned}
$$

Now the equilibrium conditions provided in (39) and (40) can be employed, from which we get (as might be expected):

$$
\varepsilon_{1}=\varepsilon_{2}=\omega_{3}=0
$$

Thus, expression (93) can be simplified as:

$\sigma_{3 \alpha}^{+}\left(x_{1}, x_{2}\right)=-\frac{4 c_{44} D C^{*} \sigma_{3} x_{\alpha}}{\pi \sqrt{a^{2}-r^{2}}}, \alpha=1,2$

From above it follows that the problem in hand is axially symmetric.

On substitution of formulas (96) into Eqs. (81) and (82), we find:

$$
\begin{aligned}
& g_{, 3}(\mathbf{x})=\frac{2 D C^{*} \sigma_{3}}{\pi^{2} t_{3}}\left(\psi_{1}+\kappa \psi_{1,2} x_{2}-\kappa \psi_{2,2} x_{1}\right) \\
& h_{, 3}(\mathbf{x})=\frac{2 D C^{*} \sigma_{3}}{\pi^{2} t_{3}}\left(\psi_{2}+\kappa \psi_{2,2} x_{1}-\kappa \psi_{1,1} x_{2}\right)
\end{aligned}
$$

Here $\psi_{\alpha}, \alpha=1,2$ are the simple-layer potentials defined by:

$\psi_{\alpha}(\mathbf{x})=\iint_{S} \frac{\xi_{\alpha} d \xi_{1} d \xi_{2}}{|\mathbf{x}-\xi| \sqrt{a^{2}-\left(\xi_{1}\right)^{2}-\left(\xi_{2}\right)^{2}}}$

They can be expressed in elementary functions by utilizing the results of Fabrikant $(1989,1991)$ as: 
$\psi_{\alpha}(\mathbf{x})=\pi x_{\alpha}\left[\sin ^{-1}\left(\frac{a}{I_{2}}\right)-\frac{a \sqrt{\left(I_{2}\right)^{2}-a^{2}}}{\left(I_{2}\right)^{2}}\right]$

where $I_{2}=0,5\left[\sqrt{(r+a)^{2}+\left(x_{3}\right)^{2}}+\sqrt{(r-a)^{2}+\left(x_{3}\right)^{2}}\right]$.

Having the above explicit expressions, a complete solution to the problem under study is available. Since the resulting formulas are lengthy, we omit them here to save the space of the paper. To investigate the singular behavior of anticrack-border stresses, however, the shear radial and normal stresses in the plane $x_{3}=0^{\mp}$ are calculated as follows:

$\sigma_{3 r}\left(r, 0^{ \pm}\right)=\left\{\begin{array}{lc}\mp \frac{\beta_{r} \sigma_{3} r}{\pi \sqrt{a^{2}-r^{2}}}, & 0 \leq r<a \\ 0, & r>a\end{array}\right.$

$\sigma_{33}\left(r, 0^{ \pm}\right)= \begin{cases}-\frac{1}{2} \beta_{3} \sigma_{3}, & 0 \leq r< \\ \frac{\beta_{3} \sigma_{3}}{\pi}\left[\frac{a}{\sqrt{r^{2}-a^{2}}}-\sin ^{-1}\left(\frac{a}{r}\right)\right], & r>a\end{cases}$

in which $\beta_{r}, \beta_{3}$ are given by:

$$
\begin{aligned}
& \beta_{r}=\frac{\left.4 c_{13} \sqrt{c_{11} c_{44}\left[c_{11} c_{33}-c_{13}\left(2 c_{44}+c_{13}\right)+2 c_{44} \sqrt{c_{11} c_{33}}\right.}\right]}{\left[c_{33}\left(c_{11}+c_{12}\right)-2\left(c_{13}\right)^{2}\right]\left(\sqrt{c_{11} c_{33}}+c_{44}\right)} \\
& \beta_{3}=\frac{4 c_{13} c_{44}\left(\sqrt{c_{11} c_{33}}-c_{13}\right)}{\left[c_{33}\left(c_{11}+c_{12}\right)-2\left(c_{13}\right)^{2}\right]\left(\sqrt{c_{11} c_{33}}+c_{44}\right)}
\end{aligned}
$$

These results reveal that the stresses near the anticrack front $r=a$ have the classical singularity $r^{-1 / 2}$ as in the fracture mechanics of conventional elastic materials. Strictly speaking, singularities in $\sigma_{3 r}$ occur at the points on the edge of the disc where $r=a^{-}$, and in $\sigma_{33}$ - at the points exterior to the disc where $r=a^{+}$. It indicates that there are two major mechanisms controlling the material cracking around the inclusion front:

- exfoliation of the material from the surface of the inclusion described by the stress singularity coefficients:

$$
S_{\mathrm{II}}^{ \pm}=\lim _{r \rightarrow a^{-}} \sqrt{2 \pi(a-r)} \sigma_{3 r}\left(r, 0^{ \pm}\right)=\mp \beta_{r} \sigma_{3} \sqrt{a / \pi}
$$

- mode I fracture in the immediate vicinity of the edge of the disc characterized by the stress intensity factor:

$$
K_{\mathrm{I}}=\lim _{r \rightarrow a^{+}} \sqrt{2 \pi(r-a)} \sigma_{33}(r, 0)=\beta_{3} \sigma_{3} \sqrt{a / \pi}
$$

The above-mentioned parameters can be used in conjunction with a suitable failure criterion.

Finally, in the special case of isotropy (see (16)), we arrive at the solution in which the constants $\beta_{r}, \beta_{3}$ become:

$$
\begin{aligned}
& \beta_{r}=\frac{4 \lambda(\lambda+2 \mu)}{(\lambda+3 \mu)(3 \lambda+2 \mu)}=\frac{8 \nu(1-\nu)}{(1+\nu)(3-4 \nu)} \\
& \beta_{3}=\frac{4 \lambda \mu}{(\lambda+3 \mu)(3 \lambda+2 \mu)}=\frac{4 \nu(1-2 \nu)}{(1+\nu)(3-4 \nu)}
\end{aligned}
$$

\section{CONCLUSIONS}

In the present paper we have studied the elastostatic threedimensional problem of an anticrack of arbitrary shape embedded in a transversely isotropic space and subjected to external loads. Using the method of potential functions, the mixed boundary-value problems in the antisymmetric and symmetric statement have been reduced to some mixed problems of potential theory. Further, the governing boundary integral equations were obtained with the unknown stress jumps across the rigid inclusion. As an illustration, a closed-form solution was given and discussed for a circular rigid inclusion subjected to normal tension at infinity. The analytical expressions of stress fields in the anticrack plane show the characteristic $r^{-1 / 2}$ singular behaviour near the edge of the disc and indicate that either the matrix fractures near the inclusion according to mode I or the mechanism of shear fracture acts (separation of the material from the inclusion surface).

\section{REFERENCES}

1. Berezhnitskii L.T., Panasyuk V.V., Stashchuk N.G. (1983), The Interaction of Rigid Linear Inclusions and Cracks in a Deformable Body (in Russian), Naukova Dumka, Kiev.

2. Chaudhuri R.A. (2003), Three-dimensional asymptotic stress field in the vicinity of the circumference of a penny-shaped discontinuity, International Journal of Solids and Structures, Vol. 40, 3787-3805.

3. Chaudhuri R.A. (2012), On three-dimensional singular stress field at the front of a planar rigid inclusion (anticrack) in an orthorhombic mono-crystalline plate, International Journal of Fracture, Vol. 174, 103-126.

4. Ding H., Chen W., Zhang L. (2006), Elasticity of Transversely Isotropic Materials, Solid Mechanics and its Applications, Vol. 126, Springer, The Netherlands.

5. Erdelyi A. (1954), Tables of Integral Transforms, Vol.1, McGraw-Hill, New York.

6. Fabrikant V.I. (1989), Applications of Potential Theory in Mechanics: A Selection of New Results, Kluwer Academic Publishers, Dordrecht.

7. Fabrikant V.I. (1991), Mixed Boundary Value Problems of Potential Theory and their Applications, Kluwer Academic Publishers, Dordrecht.

8. Kaczyński A. (1993),On the three-dimensional interface crack problems in periodic two-layered composites, International Journal of Fracture, Vol. 62, 283-306.

9. Kaczyński A. (1999), Rigid sheet-like interface inclusion in an infinite bimaterial periodically layered composite, Journal of Theoretical and Applied Mechanics, Vol. 37, 81-94.

10. Kanaun S.K., Levin V.M. (2008), Self-Consistent Methods for Composites. Vol. 1: Static Problems, Solid Mechanics and its Applications, Vol. 148, Springer,The Netherlands, Dordrecht.

11. Kassir M.K., Sih G.C. (1968), Some three-dimensional inclusion problems in elasticity, International Journal of Solids and Structures, Vol. 4, 225-241.

12. Kassir M.K., Sih G.C. (1975), Three-Dimensional Crack Problems, Mechanics of Fracture 2, Noordhoof Int. Publ., Leyden.

13. Khai M.V. (1993), Two-Dimensional Integral Equations of the Newton-Potential Type and their Applications (in Russian), Naukova Dumka, Kiev.

14. Kit G.S., Khai M.V. (1989), Method of Potentials in ThreeDimensional Problems of Thermoelasticity of Bodies with Cracks (in Russian), Naukova Dumka, Kiev.

15. Mura T. (1982), Micromechanics of Defects in Solids, Martinus Nijhoff, The Hague.

in complete agreement with Kassir and Sih (1968). 
16. Panasyuk V.V., Stadnik M.M., Silovanyuk V.P. (1986), Stress Concentrations in Three-Dimensional Bodies with Thin Inclusions (in Russian), Naukova Dumka, Kiev.

17. Podil'chuk Y.N. (1997), Stress state of a transversely-isotropic body with elliptical inclusion, International Applied Mechanics, Vol. 33, 881-887.

18. Rahman M. (1999), Some problems of a rigid elliptical disc-inclusion bonded inside a transversely isotropic space, Transactions of the ASME Journal of Applied Mechanics, Vol. 66, 612-630.

19. Rahman M. (2002), A rigid elliptical disc-inclusion, in an elastic solid, subjected to a polynomial normal shift, Journal of Elasticity, Vol. 66, 207-235.

20. Rogowski B. (2006), Inclusion Problems for Anisotropic Media, Technical University of Lodz, Lodz.

21. Selvadurai A.P.S. (1982),On the interaction between an elastically embedded rigid inhomogeneity and a laterally placed concentrated force, Journal of Applied Mathematics and Physics (ZAMP), Vol. 33, 241-250.

22. Shodja H.M., Ojaghnezhad F. (2007), A general unified treatment of lamellar inhomogeneities, Engineering Fracture Mechanics, Vol. 74, 1499-1510.

23. Silovanyuk V.P. (1984), A rigid lamellar inclusion in elastic space, Materials Science, Vol. 20, 482-485.

24. Silovanyuk V.P. (2000), Fracture of Prestressed and Transversely Isotropic Bodies with Defects, National Academy of Science of Ukraine, Physico-Mechanical Institute named G.V. Karpenko, Lviv.

25. Sneddon I.N. (1972), The Use of Integral Transforms, McGraw-Hill, New York.

26. Ting T.C.T. (1996), Anisotropic Elasticity: Theory and Applications, Oxford University Press, New York.

27. Vorovich I.I., Alexandrov V. V., Babeshko V. A. (1974), Nonclassical Mixed Boundary Problems of Theory of Elasticity (in Russian), Nauka, Moscow. 